PROCEEDINGS OF THE

AMERICAN MATHEMATICAL SOCIETY

Volume 137, Number 5, May 2009, Pages 1823-1833

S 0002-9939(08)09727-X

Article electronically published on December 15, 2008

\title{
THE PROPER AND SEMI-PROPER FORCING AXIOMS FOR FORCING NOTIONS THAT PRESERVE $\aleph_{2}$ OR $\aleph_{3}$
}

\author{
JOEL DAVID HAMKINS AND THOMAS A. JOHNSTONE
}

(Communicated by Julia Knight)

\begin{abstract}
We prove that the PFA lottery preparation of a strongly unfoldable cardinal $\kappa$ under $\neg 0^{\sharp}$ forces PFA( $\aleph_{2}$-preserving), PFA( $\aleph_{3}$-preserving) and $\mathrm{PFA}_{\aleph_{2}}$, with $2^{\omega}=\kappa=\aleph_{2}$. The method adapts to semi-proper forcing, giving $\operatorname{SPFA}\left(\aleph_{2}\right.$-preserving), SPFA( $\aleph_{3}$-preserving) and $\mathrm{SPFA}_{\aleph_{2}}$ from the same hypothesis. It follows by a result of Miyamoto that the existence of a strongly unfoldable cardinal is equiconsistent with the conjunction SPFA( $\aleph_{2}$-preserving $)+$ $\operatorname{SPFA}\left(\aleph_{3}\right.$-preserving $)+\mathrm{SPFA}_{\aleph_{2}}+2^{\omega}=\aleph_{2}$. Since unfoldable cardinals are relatively weak as large cardinal notions, our summary conclusion is that in order to extract significant strength from PFA or SPFA, one must collapse $\aleph_{3}$ to $\aleph_{1}$.
\end{abstract}

A convergence of recent results has pointed at the strongly unfoldable cardinals, relatively low in the large cardinal hierarchy, as a surprisingly efficacious substitute for supercompact cardinals in several large cardinal phenomena. For example, Johnstone [Joh07, Joh], fulfilling earlier hints that a Laver-style indestructibility phenomenon may be possible, proved that any strongly unfoldable cardinal $\kappa$ can be made indestructible by all $<\kappa$-closed $\kappa$-proper forcing. In subsequent joint work $\left[\mathrm{HJ}\right.$, we extended this to indestructibility by all $<\kappa$-closed $\kappa^{+}$-preserving forcing. In this article, we adapt the construction, just as Baumgartner modified the Laver preparation Lav78 to force the Proper Forcing Axiom (PFA) from a supercompact cardinal, to force interesting fragments of PFA from an unfoldable cardinal. Succinctly, in this article we explain how to carry out the Baumgartner PFA construction using a mere strongly unfoldable cardinal in the place of his supercompact cardinal and using the PFA lottery preparation in place of his Laver-style iteration. The end result is a new relatively low upper bound on $\operatorname{PFA}\left(\aleph_{2}\right.$-preserving) and $\mathrm{PFA}\left(\aleph_{3}\right.$-preserving). The methods extend analogously to the case of semi-proper forcing and the corresponding fragments of SPFA.

This project can be viewed as a continuation of Miyamoto's construction Miy98, where he used a hypothesis equivalent to strong unfoldability to prove the relative consistency of $\mathrm{PFA}_{\mathfrak{c}}$ (see Theorem 6 below). There is also a strong affinity

Received by the editors November 20, 2007, and, in revised form, August 13, 2008.

2000 Mathematics Subject Classification. Primary 03E55, 03E40.

Key words and phrases. Forcing axiom, strongly unfoldable cardinal.

The research of the first author has been supported in part by grants from the CUNY Research Foundation and the Netherlands Organization for Scientific Research (NWO), and he is grateful to the Institute of Logic, Language and Computation at Universiteit van Amsterdam for the support of a Visiting Professorship during his 2007 sabbatical there.

Parts of this article are adapted from the second author's Ph.D. dissertation, The Graduate Center of The City University of New York, June 2007.

(C)2008 American Mathematical Society Reverts to public domain 28 years from publication 1823 
with recent work of Neeman and Schimmerling [NS08, who used the PFA lottery preparation independently, obtaining other weak fragments of PFA from weakly indescribable cardinals.

Let us quickly review the relevant concepts. The Proper Forcing Axiom PFA is the assertion that for any proper poset $\mathbb{Q}$ and every collection $\mathcal{D}$ of at most $\aleph_{1}$ many dense subsets of $\mathbb{Q}$, there is a filter $G \subseteq \mathbb{Q}$ meeting every dense set in $\mathcal{D}$. If $\Gamma$ is a class of posets, then $\operatorname{PFA}(\Gamma)$ is the corresponding assertion restricted to proper $\mathbb{Q} \in \Gamma$. We say that a forcing notion $\mathbb{Q}$ is $\delta$-preserving if it does not collapse $\delta$ as a cardinal. Thus, the axiom PFA $\left(\aleph_{2}\right.$-preserving) asserts that for every $\aleph_{2}$-preserving proper poset $\mathbb{Q}$ and every collection $\mathcal{D}$ of $\aleph_{1}$ many dense subsets of $\mathbb{Q}$, there is a filter $G \subseteq \mathbb{Q}$ meeting every element of $\mathcal{D}$. Similarly, PFA( $\aleph_{3}$-preserving) makes the corresponding assertion about proper $\aleph_{3}$-preserving forcing. (Note that with $\aleph_{3}$ preserving forcing, we do not insist that $\aleph_{3}$ is preserved as $\aleph_{3}$, only that it remains a cardinal.) Proper forcing, of course, is always $\aleph_{1}$-preserving.

If $\mathcal{A}$ is any class of partial orders, then the lottery sum $\oplus \mathcal{A}$ is the partial order $\{\langle\mathbb{Q}, q\rangle \mid q \in \mathbb{Q} \in \mathcal{A}\} \cup\{\mathbb{1}\}$, ordered with $\mathbb{1}$ above everything and $\langle\mathbb{Q}, q\rangle \leq\langle\mathbb{P}, p\rangle$ if and only if $\mathbb{Q}=\mathbb{P}$ and $q \leq \mathbb{Q} p$. Forcing with $\oplus \mathcal{A}$ amounts to choosing a winning poset from $\mathcal{A}$ and then forcing with it. This is also commonly known as side-by-side forcing and is forcing equivalent to the Boolean product (without omitting 0) of the corresponding Boolean algebras. We use the notation $f: X \rightarrow Y$ for partial functions, to indicate that $\operatorname{dom}(f) \subseteq X$ and $\operatorname{ran}(f) \subseteq Y$. We use $\mathrm{ZFC}^{-}$to denote the theory consisting of all the usual ZFC axioms except the Power Set axiom. The lottery preparation of [Ham00 is the Easton support iteration of suitably large lottery sums of progressively closed forcing, the point being that by allowing the filter generically to select which forcing is performed at each stage, one avoids the need for a Laver function. Just as Baumgartner adapted the Laver preparation to the case of proper forcing, here we adapt the lottery preparation.

Definition 1. The PFA lottery preparation of a cardinal $\kappa$, relative to the function $f: \kappa \rightarrow \kappa$, is the countable support $\kappa$-iteration, which forces at stages $\gamma \in \operatorname{dom}(f)$ with the lottery sum of all proper forcing $\mathbb{Q}$ in $V\left[G_{\gamma}\right]$ having hereditary size at most $f(\gamma)$.

The PFA lottery preparation was introduced in Johnstone's dissertation Joh07 and later used independently by Neeman and Schimmerling in NS08. The PFA lottery preparation works best when $f$ exhibits certain fast growth behavior, such as the Menas property, discussed below, and we will only perform the preparation with respect to such functions. The PFA lottery preparation of a supercompact cardinal forces PFA (see Theorem 12), but the point of this article is to use a much smaller cardinal, a strongly unfoldable cardinal, to achieve interesting fragments of PFA.

So let us turn now to the unfoldable and strongly unfoldable cardinals, introduced by Villaveces Vil98. An inaccessible cardinal $\kappa$ is unfoldable if for every ordinal $\theta$ it is $\theta$-unfoldable, meaning that for every transitive set $M$ of size $\kappa$ with $\kappa \in M$ there is a transitive set $N$ and an elementary embedding $j: M \rightarrow N$ with critical point $\kappa$ and $j(\kappa) \geq \theta$. An inaccessible cardinal $\kappa$ is strongly unfoldable if for every ordinal $\theta$ it is $\theta$-strongly unfoldable, meaning that for every transitive set $M \models \mathrm{ZFC}^{-}$of size $\kappa$ with $\kappa \in M$ and $M^{<\kappa} \subseteq M$ there is a transitive set $N$ and an elementary embedding $j: M \rightarrow N$ with critical point $\kappa$ such that $j(\kappa) \geq \theta$ and $V_{\theta} \subseteq N$. We refer to such $M$ as the $\kappa$-models of set theory. The strongly unfoldable cardinals were 
introduced independently by Miyamoto Miy98 as the $H_{\kappa^{+}}$-reflecting cardinals, an equivalent characterization. The unfoldable and strongly unfoldable cardinals lie relatively low in the large cardinal hierarchy, somewhat above the weakly compact cardinals, and they relativize to $L$ in the sense that every unfoldable cardinal is unfoldable in $L$ and in fact strongly unfoldable there, for in $L$ the two notions coincide. For this reason, the notions of unfoldability and strong unfoldability, although not equivalent, have the same consistency strength, bounded below by the totally indescribable cardinals and above by the subtle cardinals.

Although the strongly unfoldable cardinals are defined to exhibit a miniature form of strongness, they are also known to exhibit a supercompactness nature. Specifically, [DH06 Lemma 5] shows that an inaccessible cardinal $\kappa$ is $(\theta+1)$ strongly unfoldable $(\kappa \leq \theta)$ if and only if for every $\kappa$-model $M$ there is a transitive set $N$ and an embedding $j: M \rightarrow N$ with critical point $\kappa$, such that $j(\kappa)>\theta$ and $N^{\beth_{\theta}} \subseteq N$ and $|N|=\beth_{\theta+1}$. A similar observation is made in Miy98. In particular, since $M$ and hence $j$ have size $\kappa$, such embeddings have both $M$ and $j$ as elements of $N$, a feature we call the Hauser property, since this characterization is proved by adapting the Hauser method Hau91 from the indescribable cardinal context. This supercompactness-like nature of the unfoldability embedding allows us to borrow techniques for strong unfoldability from the supercompact cardinals.

The PFA lottery preparation will work best when $f$ exhibits a certain fastgrowing behavior called the Menas property, defined for various large cardinals to ensure for suitable large cardinal embeddings $j: M \rightarrow N$ that $j(f)(\kappa)$ can be made as large as desired. The importance of this is that if $\mathbb{P}$ is the PFA lottery preparation of $\kappa$ in $M$, then the stage $\kappa$ lottery of $j(\mathbb{P})$ is expansive enough to include whatever forcing $\dot{\mathbb{Q}} \in N$ we had desired to include within it. Below a condition in $j(\mathbb{P})$ opting for this $\dot{\mathbb{Q}}$ at stage $\kappa$, therefore, the forcing $j(\mathbb{P})$ factors as $\mathbb{P} * \dot{\mathbb{Q}} * \mathbb{P}_{\text {tail }}$, where $\mathbb{P}_{\text {tail }}$ is the forcing beyond stage $\kappa$. This ability to factor $j(\mathbb{P})$ so that $\mathbb{Q}$ appears explicitly at stage $\kappa$ is the key technique by which the lottery preparation avoids the need for Laver functions. Indeed, the cardinals with which we are concerned - strongly unfoldable, unfoldable, indescribable and weakly compact cardinals - may have no Laver functions, because [DH06] shows that even $\nabla_{\kappa}(\mathrm{REG})$ may fail at them. Nevertheless, Ham shows that one can add Laver functions generically. In the particular case of a strongly unfoldable cardinal $\kappa$, we say that $f$ has the strong unfoldability Menas property if for every $\kappa$-model $M$ having $f \in M$ there is an embedding $j: M \rightarrow N$ with $\operatorname{cp}(j)=\kappa$ and $V_{\theta} \subseteq N$, and $j(f)(\kappa) \geq \theta$. (The terminology arises from Menas' prescient treatment Men74] of strongly compact cardinals.) It is not difficult to check that one can carry such a function through [DH06, Lemma 5] in order to obtain embeddings $j: M \rightarrow N$ with the Hauser property $j \in N$ as well as the Menas property $j(f)(\kappa) \geq \theta$.

Johnstone [Joh07. observed that every strongly unfoldable cardinal has a function exhibiting the strong unfoldability Menas property. Specifically, consider the failure-of-strong-unfoldability function $f: \kappa \rightarrow \kappa$, for a strongly unfoldable cardinal $\kappa$, defined by $f(\gamma)=\theta$ when $\gamma$ is an inaccessible non-strongly unfoldable cardinal below $\kappa$ and $\theta$ is least such that $\gamma$ is not $\theta$-strongly unfoldable. Because one can always find $\theta$-strong unfoldability embeddings $j: M \rightarrow N$ for which $\kappa$ is not strongly unfoldable in $N$, while $V_{\theta} \subseteq N$ ensures that $\kappa$ is at least $<\theta$-strongly unfoldable in $N$, it follows that $j(f)(\kappa) \geq \theta$, as desired. Subsequently, we shall only consider the PFA lottery preparation with respect to functions having the Menas property. 
Finally, we are ready to prove the first main theorem, obtaining a fragment of PFA from unfoldability. Since every unfoldable cardinal is strongly unfoldable in $L$, the hypothesis of the next theorem is equiconsistent with the existence of an unfoldable cardinal. This theorem answers Question 59 of [Joh07].

Theorem 2. If $\kappa$ is strongly unfoldable and $V=L$, then the PFA lottery preparation of $\kappa$ forces $\mathrm{PFA}\left(\aleph_{2}\right.$-preserving $)$ and $\mathrm{PFA}\left(\aleph_{3}\right.$-preserving $)$, with $2^{\omega}=\kappa=\aleph_{2}$.

Proof. Assume $\kappa$ is strongly unfoldable and $V=L$. Let $\mathbb{P}$ be the PFA lottery preparation of $\kappa$ defined relative to a function $f: \kappa \rightarrow \kappa$ with the Menas property, and suppose that $G \subseteq \mathbb{P}$ is $L$-generic. Since the lottery sum of proper forcing is proper, each stage of this forcing is proper and consequently the entire iteration $\mathbb{P}$ is proper. Thus, $\omega_{1}$ is preserved between $L$ and $L[G]$. If $\gamma$ is a cardinal of $L$ between $\omega_{1}$ and $\kappa$, however, then the forcing to collapse $\gamma$ to $\omega_{1}$, being countably closed and hence proper and having size less than $\kappa$, will appear unboundedly often in the lottery sums of $\mathbb{P}$; consequently, by genericity, there will be many stages at which the generic filter $G$ opts for it and so all such $\gamma$ are collapsed to $\omega_{1}$ in $L[G]$. Meanwhile, a standard $\Delta$-system argument shows that $\mathbb{P}$ has the $\kappa$-c.c., and so at the top the cardinal $\kappa$ itself is preserved, becoming $\aleph_{2}^{L[G]}$. The usual nice-name counting arguments show that the continuum $2^{\omega}$ and even $2^{\omega_{1}}$ in $L[G]$ are at most $\kappa$. Conversely, the generic filter will opt at unboundedly many stages to add a Cohen real, and so the continuum of $L[G]$ is at least $\kappa$. In summary, $L[G]$ satisfies $\kappa=\aleph_{2}=\mathfrak{c}=2^{\omega}=2^{\omega_{1}}=\mathfrak{c}^{<\mathfrak{c}}$, and $\aleph_{3}^{L[G]}=\kappa^{+L}$.

Let us show next that $L[G]$ satisfies $\operatorname{PFA}\left(\aleph_{3}\right.$-preserving). In order to do so, suppose that $\mathbb{Q}$ is an $\aleph_{3}$-preserving proper notion of forcing in $L[G]$ and $\mathcal{D}$ is a family of $\aleph_{1}$ many dense subsets of $\mathbb{Q}$. Let $\dot{\mathbb{Q}}$ be a $\mathbb{P}$-name for $\mathbb{Q}$, forced by $\mathbb{1}$ to have the properties mentioned. We will find a $\mathcal{D}$-generic filter on $\mathbb{Q}$ in $L[G]$. Let $g \subseteq \mathbb{Q}$ be $L[G]$-generic. Although $g$ may happen to collapse $\kappa=\aleph_{2}$ to $\omega_{1}$, we know that $\kappa^{+L}$ remains a cardinal in $L, L[G]$ and $L[G][g]$. Let $\lambda$ be a regular cardinal

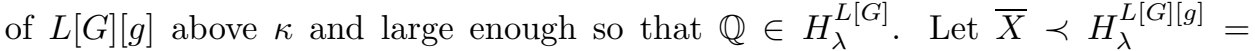
$L_{\lambda}[G][g]$ be an elementary substructure of size $|\kappa|$ in $L[G][g]$, containing the objects $\{\mathbb{P}, \kappa, \mathbb{Q}, f, \mathcal{D}, G, g\}$ and with $\kappa \subseteq \bar{X}$. Let $X=\bar{X} \cap L$, which is an elementary substructure of $H_{\lambda}^{L}=L_{\lambda}$. Since $\bar{X}$ satisfies that $G * g \subseteq \mathbb{P} * \dot{\mathbb{Q}}$ is $L$-generic, it follows for every dense set $D \subseteq \mathbb{P} * \dot{\mathbb{Q}}$ with $D \in X$ that $\bar{X}$ has witnessing elements in $G * g \cap D \cap \bar{X}$. Since $D \subseteq L$, these witnesses must be in $\bar{X} \cap L$ and hence in $X$. Thus, $G * g \cap D \cap X \neq \emptyset$ for every dense set $D \in X$, which is to say that the filter $G * g$ is $X$-generic. It follows that $\bar{X}=X[G][g]$ and $g$ is $X[G]$-generic. In particular, we have

$$
g \cap D \cap X[G] \neq \emptyset
$$

for every dense set $D \subseteq \mathbb{Q}$ with $D \in X[G]$. Since $\mathcal{D} \in X[G]$ has size $\omega_{1}$, it follows that $\mathcal{D} \subseteq X[G]$, and this consequently includes every $D \in \mathcal{D}$. Although $X \subseteq L$, we do not know that $X \in L$. Since $\bar{X}$ had size $|\kappa|$ in $L[G][g]$, it follows that $X$ also has size $|\kappa|$ in $L[G][g]$.

We now make the key step of the argument. Since $0^{\sharp}$ cannot be added by set forcing over $L$, we may apply Jensen's Covering Theorem in $L[G][g]$ to find a set $Y \in L$ with $X \subseteq Y$ and $|X|=|Y|$ in $L[G][g]$. Since $\kappa^{+}$was preserved as a cardinal, it follows that $Y$ has cardinality $\kappa$ in $L$. We may assume $Y \subseteq H_{\lambda}^{L}$. Indeed, since $\kappa$ is inaccessible in $L$, we may further assume that $Y$ is closed under Skolem functions and $<\kappa$-sequences, so that $Y \prec H_{\lambda}^{L}$ and $Y^{<\kappa} \subseteq Y$ in $L$. Since 
$\mathbb{P} \subseteq X \subseteq Y \subseteq L$ and $G \subseteq \mathbb{P}$ is $L$-generic, it follows that $G$ is also $Y$-generic, and that $Y[G] \prec H_{\lambda}^{L}[G]$. Although $g \subseteq \mathbb{Q}$ is $X[G]$-generic, we do not know that $g$ is $Y[G]$-generic. Nevertheless, the degree of genericity expressed in the displayed equation of the previous paragraph will be sufficient for our purposes.

Let $\pi: Y \cong M$ be the Mostowski collapse of $Y$ in $L$. Since $G \subseteq \mathbb{P}$ is $Y$-generic, this agrees with the Mostowski collapse of the extension $\pi: Y[G] \cong M[G]$. Note that $\pi$ is the identity on $\mathbb{P}, G$ and their elements because $\mathbb{P} \subseteq Y$. The structure $M$ is a transitive set model of $\mathrm{ZFC}^{-}$, with $|M|=\kappa$ and $M^{<\kappa} \subseteq M$ in $L$. In short, $M$ is a $\kappa$-model in $L$. (In fact, by the Condensation Principle, it follows that $M=L_{\eta}$ for some $\eta<\kappa^{+}$, but we will not make use of this fact.) Let $\theta$ be a large enough ordinal above $\lambda$ so that the properness of $\mathbb{Q}$ is verified in $V_{\theta}^{L}[G]$. Since $M$ is a $\kappa$-model in $L$ and $\kappa$ is strongly unfoldable there, there is a $\theta$-strong unfoldability embedding $j: M \rightarrow N$ with the Hauser property, so that $V_{\theta}^{L} \subseteq N$ and $M$ and $j$ are elements of $N$, and the Menas property, so that $j(f)(\kappa) \geq \theta$. Consider the forcing $j(\mathbb{P})$ in $N$, which is the PFA lottery preparation of $j(\kappa)$ defined with respect to $j(f)$. Up to stage $\kappa$, this agrees with $\mathbb{P}$, and $G \subseteq \mathbb{P}$ is $N$-generic. Since $V_{\theta}^{L} \subseteq N \in L$, it follows that $V_{\theta}^{L[G]}=V_{\theta}^{N[G]}$, and consequently, $\mathbb{Q}$ is in $N[G]$ and proper there, since $\theta$ was chosen to be large enough to witness this. Since $j(f)(\kappa) \geq \theta$, it follows that $\mathbb{Q}$ appears in the stage $\kappa$ lottery of $j(\mathbb{P})$ in $N[G]$. Below a condition opting for $\mathbb{Q}$ in this lottery sum, therefore, we may factor the forcing $j(\mathbb{P})$ as $\mathbb{P} * \mathbb{Q} * \mathbb{P}_{\text {tail }}$, where $\mathbb{P}_{\text {tail }}$ is the forcing after stage $\kappa$. Force to add an $L[G][g]$-generic filter $G_{\text {tail }} \subseteq \mathbb{P}_{\text {tail }}$. Thus, $G * g * G_{\text {tail }} \subseteq j(\mathbb{P})$ is $N$-generic, and in $L[G][g]\left[G_{\text {tail }}\right]$ we may lift the embedding to $j: M[G] \rightarrow N[j(G)]$, with $j(G)=G * g * G_{\text {tail }}$.

Let $\mathbb{Q}_{0}=\pi(\mathbb{Q}), \mathcal{D}_{0}=\pi(\mathcal{D})$ and $g_{0}=\pi "(g \cap X[G])$. (It would also seem natural to use $\pi$ " $(g \cap Y[G])$ here, but there is a subtle question about whether this is actually directed and consequently whether it generates a filter; so we use $\pi "(g \cap X[G])$ to skirt this issue.) Since $V_{\theta}^{L} \subseteq N$, it follows that $Y$ and $M$ are in $N$ and that $\bar{X}$ exists in $N[G][g]$. From this, it follows that $Y[G], M[G]$ and $\pi$ are in $N[G]$ and that $X[G]$ exists in $N[G][g]$. Since $j \uparrow M$ was in $N$, and the lift of $j$ to $j: M[G] \rightarrow N[j(G)]$ is defined by $j: \tau_{G} \mapsto j(\tau)_{j(G)}$, it follows that the lift of $j$ is in $N[j(G)]$. Thus, $N[j(G)]$ is able to take the collapse of $\mathbb{Q}$ to $\mathbb{Q}_{0}$, take the image of $g \cap X[G]$ to $g_{0}$ under this collapse and then the image $j " g_{0}$. We conclude, consequently, that $j " g_{0}$ is an element of $N[j(G)]$. Note that $g \cap X[G]$ is a directed subset of $\mathbb{Q} \cap X[G]$ since $g$ is an $X[G]$-generic filter on $\mathbb{Q}$. It follows that $g_{0}$ is a directed subset of $\mathbb{Q}_{0}$, and consequently $j$ " $g_{0}$ is a directed subset of $j\left(\mathbb{Q}_{0}\right)$. It therefore generates a filter on $j\left(\mathbb{Q}_{0}\right)$. Furthermore, since $\mathcal{D}$ has size $\aleph_{1}$ in $X[G]$, it follows that $\mathcal{D} \subseteq Y[G]$, and so $\mathcal{D}_{0}=\pi(\mathcal{D})=\pi " \mathcal{D}$ also has size $\aleph_{1}$ and consequently $j\left(\mathcal{D}_{0}\right)=j " \mathcal{D}_{0}$. Thus, every element of $j\left(\mathcal{D}_{0}\right)$ has the form $j(\pi(D))$ for some $D \in \mathcal{D}$. Since we established $g \cap D \cap X[G] \neq \emptyset$ in the displayed equation above, it follows that $g_{0} \cap \pi(D) \neq \emptyset$ and consequently $\left(j " g_{0}\right) \cap j(\pi(D)) \neq \emptyset$. In short, the filter on $j\left(\mathbb{Q}_{0}\right)$ generated by $j$ " $g_{0}$ in $N[j(G)]$ meets every dense set in $j\left(\mathcal{D}_{0}\right)$. Since $j: M[G] \rightarrow N[j(G)]$ is elementary, we conclude that $M[G]$ has a filter on $\mathbb{Q}_{0}$ meeting every dense set in $\mathcal{D}_{0}$. Since $Y[G] \cong M[G]$ via $\pi$, it follows that $Y[G]$ has a filter on $\mathbb{Q}$ meeting every dense set in $\mathcal{D}$. Since $Y[G] \prec H_{\lambda}^{L}[G]$, it follows that $L[G]$ has a filter on $\mathbb{Q}$ meeting every dense set in $\mathcal{D}$, as desired for this fragment of PFA. Thus, we have established $\mathrm{PFA}\left(\aleph_{3}\right.$-preserving) in $L[G]$, as desired. 
The case of PFA( $\aleph_{2}$-preserving) requires only a subtle change in the argument. If in $L[G]$ we have that $\mathbb{Q}$ is proper and $\aleph_{2}$-preserving, but not $\aleph_{3}$-preserving, then we choose $\bar{X} \prec H_{\lambda}^{L[G][g]}$ of size $\aleph_{1}$, rather than size $\kappa=\aleph_{2}$. As before, we insist that $\bar{X}$ contains the objects $\{\mathbb{P}, \kappa, \mathbb{Q}, f, \mathcal{D}, G, g\}$, but now we only request that $\aleph_{1} \subseteq \bar{X}$, rather than $\kappa \subseteq \bar{X}$. It remains true that $G * g$ is $X$-generic, where $X=\bar{X} \cap L \prec H_{\lambda}^{L}$, and that $\bar{X}=X[G * g]$, but now $X$ has size $\aleph_{1}$ in $L[G][g]$. By covering, there is $X^{*} \in L$ with $X \subseteq X^{*} \subseteq H_{\lambda}^{L}$ and $\left|X^{*}\right|^{L[G][g]}=\aleph_{1}$. Since $\kappa$ was not collapsed in $L[G][g]$, it follows that $X^{*}$ has size less than $\kappa$ in $L$. Now, by further closing under Skolem functions and $<\kappa$-sequences, we may find $Y \in L$ with $X^{*} \subseteq Y \prec H_{\lambda}^{L}$ and $Y^{<\kappa} \subseteq Y$ in $L$, and $|Y|^{L}=\kappa$. We may now continue with the argument above. Since $\mathbb{P} \subseteq Y$, we get that $G \subseteq \mathbb{P}$ is $Y$-generic, and although $g \subseteq \mathbb{Q} \in Y[G]$ may not be $Y[G]$-generic, we know from the fact that $g$ was $X[G]$-generic that $g \cap D \cap X[G] \neq \emptyset$ for all $D \in X[G]$, and consequently for all $D \in \mathcal{D}$. The rest of the argument proceeds as before. We conclude that $L[G]$ satisfies $\mathrm{PFA}\left(\aleph_{2}\right.$-preserving) as well.

The argument generalizes beyond $V=L$ to the following.

Theorem 3. If $\kappa$ is strongly unfoldable and $0^{\sharp}$ does not exist, then the PFA lottery preparation of $\kappa$ forces $\mathrm{PFA}\left(\aleph_{2}\right.$-preserving $)$ and $\mathrm{PFA}\left(\aleph_{3}\right.$-preserving $)$, with $2^{\omega}=\kappa=$ $\aleph_{2}$.

Proof. The point is that if $0^{\sharp}$ does not exist, then Jensen's covering theorem relativizes and we get covering for $V \subseteq V[G][g]$ just as we did above for $L \subseteq L[G][g]$, and the argument proceeds just as above, but replacing $L$ with $V$. So the structure $X=\bar{X} \cap V$ is covered by an element $Y \in V$ of size $\kappa$, which we may assume has $Y \prec H_{\lambda}^{V}$ and $Y^{<\kappa} \subseteq Y$ in $V$.

In the case that $0^{\sharp}$ exists and all other suitable covering principles fail, then the key step of the argument appears to break down. Nevertheless, by restricting the class of forcing somewhat, we can still retain a significant fragment of PFA. We say that a forcing notion $\mathbb{Q}$ satisfies $\delta$-covering if any new set of ordinals of size less than $\delta$ added by $\mathbb{Q}$ is covered by a ground model set of size less than $\delta$ in the ground model. That is, for every $A \subseteq$ ORD in the corresponding forcing extension $V[G]$ with $|A|^{V[G]}<\delta$, there is $B \in V$ with $A \subseteq B$ and $|B|^{V}<\delta$. Note that any $\delta$-covering forcing is necessarily $\delta$-preserving. The principle $\mathrm{PFA}\left(\aleph_{2}\right.$-covering $)$ is the assertion that for any $\aleph_{2}$-covering proper forcing notion $\mathbb{Q}$ and any family $\mathcal{D}$ of $\aleph_{1}$ many dense sets of $\mathbb{Q}$, there is a filter on $\mathbb{Q}$ meeting all the dense sets in $\mathcal{D}$. We define $\mathrm{PFA}\left(\aleph_{3}\right.$-covering) similarly.

Theorem 4. If $\kappa$ is strongly unfoldable, then regardless of whether $0^{\sharp}$ exists or not, the PFA lottery preparation of $\kappa$ forces $\mathrm{PFA}\left(\aleph_{2}\right.$-covering $)$ and $\mathrm{PFA}\left(\aleph_{3}\right.$-covering $)$, with $2^{\omega}=\kappa=\aleph_{2}$.

Proof. We carry out the construction of Theorem 2, but rather than using Jensen's Covering Theorem at the key step, we instead use the fact that $\mathbb{Q}$ has the appropriate covering property over $V[G]$. Since $\mathbb{P}$ is $\kappa$-c.c., it follows that $\mathbb{P} * \mathbb{Q}$ has the analogous covering property over $V$, and this is exactly what is needed to find the desired covering set $Y \in V$ with $X \subseteq Y$ and $|Y|^{V}=\kappa$. The rest of the argument proceeds just as before. 
Pushing the argument a bit harder, we can unify and generalize the two principles and achieve the PFA for forcing $\mathbb{Q}$ with what we call the $\left(\aleph_{2}, \aleph_{3}\right)$-cover property, meaning that after forcing with $\mathbb{Q}$, every set of ordinals of size less than $\aleph_{2}^{V}$ is covered by a ground model set of size less than $\aleph_{3}^{V}$ in $V$. We view Theorem 4 as a generalization of Theorems 2 and 3 , because when $0^{\sharp}$ does not exist, then a forcing notion $\mathbb{Q}$ has $\aleph_{2}$-covering if and only if it is $\aleph_{2}$-preserving, and has $\aleph_{3^{-}}$ covering if and only if it is $\aleph_{3}$-preserving, since in each case the covering part follows for free from the Covering Theorem. Theorem 4 generalizes [Joh07, Theorem 57], which used the PFA lottery preparation of a strongly unfoldable cardinal to obtain PFA( $\aleph_{2}$-proper $)$, since every $\aleph_{2}$-proper notion of forcing also has $\aleph_{3}$-covering.

Next, we show how the same PFA lottery preparation extension exhibits another PFA principle, introduced by Goldstern and Shelah GS95 and investigated by Miyamoto Miy98.

Definition 5. The Proper Forcing Axiom $\mathrm{PFA}_{\delta}$ is the assertion that for any proper complete Boolean algebra $\mathbb{Q}$ and any collection $\mathcal{D}$ of at most $\aleph_{1}$ many maximal antichains, each of size at most $\delta$, there is a filter on $\mathbb{Q}$ meeting all maximal antichains in $\mathcal{D}$.

One can equivalently express $\mathrm{PFA}_{\delta}$ in terms of arbitrary proper posets, rather than only proper complete Boolean algebras, but we caution the reader in this case that special care is required when doing so. In order to have an equivalent principle, the conclusion of $\mathrm{PFA}_{\delta}$ in the case of proper posets $\mathbb{Q}$ should be only that there is a centered subset of $\mathbb{Q}$ meeting the antichains, rather than a filter. A subset $C$ of a poset $\mathbb{Q}$ is centered if every finite subset of $C$ has a lower bound in $\mathbb{Q}$, but not necessarily in $C$. Every filter, of course, is centered, but not every centered set in a poset generates a filter (although it does in every Boolean algebra, and more generally, in any poset with the property that any two compatible elements have a greatest lower bound). It is not clear that one should expect filters with $\mathrm{PFA}_{\delta}$ for posets as opposed to centered sets, because if $\mathbb{Q}$ is a poset and $F$ is a filter on its completion $\mathbb{B}$ as a complete Boolean algebra, then although the restriction $F \cap \mathbb{Q}$ is centered in $\mathbb{Q}$, it is not necessarily a filter in $\mathbb{Q}$ and it may not even be directed. We find it natural anyway in the $\mathrm{PFA}_{\delta}$ context to prefer complete Boolean algebras, since they have a richer collection of small antichains; after all, every poset is forcing-equivalent to a poset having no small maximal antichains at all (consider the lottery sum of the poset with itself $\delta^{+}$many times, but without any $\mathbb{1}$ ), thereby trivializing these instances of $\mathrm{PFA}_{\delta}$. For all these reasons, we take $\mathrm{PFA}_{\delta}$ as defined above, using complete Boolean algebras and filters.

We point out that our model also exhibits $\mathrm{PFA}_{\mathfrak{c}}$.

Theorem 6. If $\kappa$ is strongly unfoldable, then the PFA lottery preparation of $\kappa$ forces $\mathrm{PFA}_{\mathfrak{c}}$.

Proof. The main result of Miyamoto Miy98 also provides a model of $\mathrm{PFA}_{\mathfrak{c}}$ from a strongly unfoldable cardinal $\kappa$ (equivalent to $\kappa$ being $H_{\kappa^{+}-\text {reflecting) by first }}$ forcing to add an appropriate Laver function and then carrying out a Laver-style proper iteration in place of our PFA lottery preparation. Suppose that $\kappa$ is strongly unfoldable and that $G \subseteq \mathbb{P}$ is $V$-generic for the PFA lottery preparation of $\kappa$ with respect to a function $f: \kappa \rightarrow \kappa$ with the Menas property. We aim to show that $\mathrm{PFA}_{\mathfrak{c}}$ holds in $V[G]$. As in Theorem 2, we know that $V[G]$ satisfies $\kappa=\mathfrak{c}=\aleph_{2}=$ $2^{\omega}=2^{\omega_{1}}=\mathfrak{c}^{<\mathfrak{c}}$. Suppose that $\mathbb{Q}$ is a proper complete Boolean algebra in $V[G]$. 
(We would actually only need that $\mathbb{Q}$ is a proper poset which contains for any two compatible elements a unique greatest lower bound.) Let $\mathcal{D}$ be a family of $\aleph_{1}$ many maximal antichains in $\mathbb{Q}$, each of size at most $\kappa$. We do not assume here that $\mathbb{Q}$ preserves $\aleph_{2}$ or $\aleph_{3}$ or has any covering property. Let $\dot{\mathbb{Q}}$ and $\dot{\mathcal{D}}$ be $\mathbb{P}$-names for $\mathbb{Q}$ and $\mathcal{D}$, forced by $\mathbb{1}$ to have the properties we have mentioned. Pick $\lambda$ sufficiently large that $\dot{\mathbb{Q}} \in H_{\lambda}$. In $V$, choose $X \prec H_{\lambda}$ with $|X|=\kappa$ and $X^{<\kappa} \subseteq X$, such that $\mathbb{P}, \dot{\mathbb{Q}}, \dot{\mathcal{D}}$ and $f$ are all in $X$. Since $\mathbb{P} \subseteq X$, it follows that $G$ is $X$-generic and $X[G] \prec H_{\lambda}[G]=H_{\lambda}^{V[G]}$. Let $g \subseteq \mathbb{Q}$ be $V[G]$-generic. Although we have no reason to suppose that $g$ is $X[G]$-generic, we do know that $\mathcal{D} \in X[G]$ and $\mathcal{D} \subseteq X[G]$, and since every maximal antichain $A \in \mathcal{D}$ has size $\kappa$, it follows also that $A \subseteq X[G]$ as well. Thus, since $g \cap A \neq \emptyset$ for such $A$, it follows that

$$
g \cap A \cap X[G] \neq \emptyset .
$$

As in Theorem 2, this will be sufficient genericity for our purposes. Specifically, let $\pi: X \cong M$ be the Mostowski collapse of $X$, so that $M$ is a $\kappa$-model in $V$. Since $G$ was $X$-generic, it follows that this agrees with the Mostowski collapse of the extension $\pi: X[G] \cong M[G]$. Choose $\theta>\lambda$ large enough that $V_{\theta}[G]$ can verify the properness of $\mathbb{Q}$ in $V[G]$. Since $\kappa$ is strongly unfoldable in $V$, there is a $\theta$-strong unfoldability embedding $j: M \rightarrow N$, with $V_{\theta} \subseteq N$ and $j(f)(\kappa) \geq \theta$, as well as $M, j \in N$. As before, $j(\mathbb{P})$ is the PFA lottery preparation of $j(\kappa)$ relative to $j(f)$, which agrees with $\mathbb{P}$ up to stage $\kappa$. Since $V_{\theta}[G] \subseteq N[G]$, it follows that $\mathbb{Q}$ is proper in $N[G]$ and appears in the lottery sum at stage $\kappa$ of $j(\mathbb{P})$. Below a condition opting for $\mathbb{Q}$ at this stage, we may factor $j(\mathbb{P})$ as $\mathbb{P} * \dot{\mathbb{Q}} * \mathbb{P}_{\text {tail }}$. Force to add a $V[G][g]$ generic filter $G_{\text {tail }} \subseteq \mathbb{P}_{\text {tail }}$ and lift the embedding to $j: M[G] \rightarrow N[j(G)]$, where $j(G)=G * g * G_{\mathrm{tail}}$.

Let $\mathbb{Q}_{0}=\pi(\mathbb{Q})$ be the image of $\mathbb{Q}$ under the Mostowski collapse $\pi$, and let $\mathcal{D}_{0}$ be the collapse of $\mathcal{D}$. Let $g_{0}=\pi "(g \cap X[G])$ be the image of $g$ under $\pi$. Since $V_{\theta} \subseteq N$, it follows that $X \in N$ and so $N[j(G)]$ is able to construct the collapse $\pi$ of $X[G]$ and consequently $\mathbb{Q}_{0}$ and $g_{0}$ are both in $N[j(G)]$. Since $j\lceil M$ was in $N$, the model $N[j(G)]$ is able to construct the lift of $j$ to $M[G]$. Therefore, the object $j " g_{0}$ is in $N[j(G)]$. Since $X[G]$ is closed under meets in $\mathbb{Q}$ and since $g$ is a filter on $\mathbb{Q}$, it follows that $g \cap X[G]$ is a directed subset of $\mathbb{Q} \cap X[G]$. The set $g_{0}$ is therefore a directed subset of $\mathbb{Q}_{0}$ and consequently $j$ " $g_{0}$ is a directed subset of $j\left(\mathbb{Q}_{0}\right)$. Since $g \cap A \cap X[G] \neq \emptyset$, it follows that $g_{0} \cap \pi(A) \neq \emptyset$ and consequently $\left(j " g_{0}\right) \cap j(\pi(A)) \neq \emptyset$ for every $A \in \mathcal{D}$. Since $\mathcal{D}$ has size $\omega_{1}$, it follows that every element of $j\left(\mathcal{D}_{0}\right)$ has the form $j(\pi(A))$ for some $A \in \mathcal{D}$. In summary, the filter on $j\left(\mathbb{Q}_{0}\right)$ generated by $j " g_{0}$ in $N[j(G)]$ meets every antichain in $j\left(\mathcal{D}_{0}\right)$. By the elementarity of $j: M[G] \rightarrow N[j(G)]$, it follows that $M[G]$ has a filter on $\mathbb{Q}_{0}$ meeting every antichain in $\mathcal{D}_{0}$, and so $X[G]$ has a filter on $\mathbb{Q}$ meeting every antichain in $\mathcal{D}$, meaning that $V[G]$ has a filter on $\mathbb{Q}$ meeting every antichain in $\mathcal{D}$, as desired. So $V[G] \models \mathrm{PFA}_{\kappa}$, as we claimed.

We may assemble the previous theorems into an equiconsistency:

Corollary 7. The following are equiconsistent over ZFC:

(1) There is an unfoldable cardinal $\kappa$.

(2) $\operatorname{PFA}\left(\aleph_{2}\right.$-preserving $)+\operatorname{PFA}\left(\aleph_{3}\right.$-preserving $)+\mathrm{PFA}_{\aleph_{2}}+2^{\omega}=\aleph_{2}$.

(3) $\mathrm{PFA}_{\mathfrak{c}}$. 
Proof. The equiconsistency of statements 1 and 3 is essentially due to Miyamoto Miy98, since he proved that $\mathrm{PFA}_{\mathfrak{c}}$ is equiconsistent with the existence of a strongly unfoldable cardinal, which by Villaveces Vil98 is equiconsistent with the existence of an unfoldable cardinal, because every unfoldable cardinal is strongly unfoldable in $L$. We have established that the consistency of statement 1 implies that of statement 2 via the PFA lottery preparation, and clearly statement 2 implies statement 3.

The natural question arises as to the relationship between the three principles.

Question 8. Do any of the principles PFA( $\aleph_{2}$-preserving), PFA( $\aleph_{3}$-preserving) or $\mathrm{PFA}_{\mathfrak{c}}$ imply any of the others? Are the former principles equiconsistent with the latter?

By Corollary 7 , the principle $\mathrm{PFA}_{\mathfrak{c}}$ has sufficient consistency strength to imply the others, but we do not yet have an outright implication. We note that the argument of Theorem 6 was somewhat easier than that of Theorem 2 the issue of covering was avoided and we were able to work in a straightforward way with elementary substructures in the ground model. Since it was easier to establish $\mathrm{PFA}_{\mathfrak{c}}$ than $\mathrm{PFA}\left(\aleph_{2}\right.$-preserving) or PFA( $\aleph_{3}$-preserving), perhaps this indicates that these latter principles may be fully as strong as an unfoldable cardinal, just as $\mathrm{PFA}_{\mathfrak{c}}$ is.

Let us now extract a more local version of the theorem by paying careful attention to the size of $\mathbb{Q}$ required in the main arguments.

Theorem 9. If $\kappa$ is $(\theta+1)$-strongly unfoldable, with $\kappa \leq \theta$, and $0^{\sharp}$ does not exist, then the PFA lottery preparation of $\kappa$ forces the principles $\mathrm{PFA}\left(\aleph_{2}\right.$-preserving $\left.\cap V_{\theta}\right)$, $\operatorname{PFA}\left(\aleph_{3}\right.$-preserving $\left.\cap V_{\theta}\right)$ and $\operatorname{PFA}_{\mathfrak{c}}\left(V_{\theta}\right)$.

Proof. The point is that if $\mathbb{Q} \in V_{\theta}$, then having $N^{\beth_{\theta}} \subseteq N$ is sufficient in order to know that $\mathbb{Q} \in N[G]$ and $N[G] \models \mathbb{Q}$ is proper. Thus, the hypothesis of $(\theta+1)$-strong unfoldability is sufficient to handle such $\mathbb{Q}$ in $V[G]$.

Since $\kappa$ is totally indescribable if and only if it is $(\kappa+m)$-strongly unfoldable for every finite $m$, we similarly obtain the following.

Corollary 10. If $\kappa$ is totally indescribable and $0^{\sharp}$ does not exist, then the PFA lottery preparation of $\kappa$ forces $\mathrm{PFA}\left(\right.$ size $<\beth_{\omega}+\aleph_{2}$-preserving $)$ and $\mathrm{PFA}\left(\right.$ size $<\beth_{\omega}+$ $\aleph_{3}$-preserving).

One may view the recent results of Neeman and Schimmerling as further refinements of this method.

Theorem 11 (Neeman [Nee08, Neeman+Schimmerling [NS08]).

(1) $\mathrm{PFA}(\mathfrak{c}-$ linked $)$ is equiconsistent over ZFC with the existence of a $\Sigma_{1}^{2}$ indescribable cardinal.

(2) If the existence of a $\Sigma_{2}^{2}$-indescribable cardinal is consistent with ZFC, then so is $\mathrm{PFA}\left(\mathfrak{c}^{+}\right.$-c.c. $)$.

Indescribability is of course a weak form of strong unfoldability, and $\mathfrak{c}$-linked forcing is contained within $\mathfrak{c}^{+}$-c.c., and as $\mathfrak{c}=\aleph_{2}$ in their model, this forcing is contained within $\aleph_{3}$-preserving. So they attain a somewhat weaker form of PFA from a somewhat weaker large cardinal notion than we have in our main theorems above. Neeman and Schimmerling first prove that one may reduce to the case of $\mathbb{Q}$ of size at most $\mathfrak{c}^{+}$, and then perform the PFA lottery preparation and argue via the reflection form of indescribability. We expect that one could cast their argument, 
however, in terms of the indescribability embeddings of Hauser Hau91, having the form $j: M \rightarrow N$ with $V_{\kappa+1} \subseteq N, N^{\kappa} \subseteq N$ and $N$ is sufficiently correct that $\mathbb{Q}$ is in $N$ and proper there, so that it appears in the stage $\kappa$ lottery. In this situation, one could proceed as in Theorem 2, Cast in this embedding form, their argument would more closely resemble our construction and the original Baumgartner argument.

For completeness, we now describe the Baumgartner construction from a supercompact cardinal. A function $f: \kappa \rightarrow \kappa$ has the supercompactness Menas property if for every $\theta$ there is a $\theta$-supercompactness embedding $j: V \rightarrow M$, so that $\operatorname{cp}(j)=\kappa$ and $M^{\theta} \subseteq M$, such that $j(f)(\kappa) \geq \theta$. It is not difficult to construct such a function from the failure-of-supercompactness function $\gamma \mapsto$ the least $\lambda$ such that $\gamma$ is not $\lambda$-supercompact.

Theorem 12. The PFA lottery preparation of a supercompact cardinal $\kappa$, relative to a function with the supercompactness Menas property, forces PFA.

Proof. This is essentially the Baumgartner argument, but using the lottery preparation in place of his Laver-style iteration. Suppose that $G \subseteq \mathbb{P}$ is $V$-generic for the lottery preparation $\mathbb{P}$ relative to $f$, and that $\mathbb{Q}$ is proper in $V[G]$ and $\mathcal{D}$ is a family of $\aleph_{1}$ many dense subsets in $V[G]$. Choose $\theta$ large enough so that if $j: V \rightarrow M$ is a $\theta$-supercompactness embedding, then $\mathbb{Q} \in H_{\theta}^{M}[G]$ and $\mathbb{Q}$ is proper there. By using the Menas property $j(f)(\kappa) \geq \theta$, it follows that $\mathbb{Q}$ appears in the stage $\kappa$ lottery of $j(\mathbb{P})$, and so below a condition opting for $\mathbb{Q}$ in this lottery, we may factor $j(\mathbb{P})$ as $\mathbb{P} * \mathbb{Q} * \mathbb{P}_{\text {tail }}$. Let $g * G_{\text {tail }}$ be $V[G]$-generic for the forcing $\mathbb{Q} * \mathbb{P}_{\text {tail }}$, and lift the embedding to $j: V[G] \rightarrow M[j(G)]$, where $j(G)=G * g * G_{\text {tail }}$. Since $j " \theta \in M$, we may construct $j$ " $g \subseteq j(\mathbb{Q})$ in $M[j(G)]$. Since $g \subseteq \mathbb{Q}$ is a filter, it follows that $j " g$ is directed and hence generates a filter on $j(\mathbb{Q})$. Furthermore, if $D \in \mathcal{D}$, then $D \cap g \neq \emptyset$ by genericity, so $j(D) \cap j " g \neq \emptyset$ also. Since $\mathcal{D}$ has size $\aleph_{1}$, which is less than $\kappa$, it follows that $j(\mathcal{D})=j " \mathcal{D}$, and so $j " g$ meets every element of $j(\mathcal{D})$. In short, $M[j(G)]$ has a filter on $j(\mathbb{Q})$ meeting every dense set in $j(\mathcal{D})$. By elementarity, it follows that $V[G]$ has a filter on $\mathbb{Q}$ for $\mathcal{D}$, as desired.

Let us close this paper by mentioning that the theorems of this article all generalize to the case of semi-proper forcing and SPFA, by using revised countable support instead of countable support in the PFA lottery preparation. Specifically, we define the SPFA lottery preparation of $\kappa$ relative to $f: \kappa \rightarrow \kappa$ to be the revised countable support $\kappa$-iteration that at stage $\gamma$ forces with the lottery sum of all semi-proper $\mathbb{Q}$ in $H_{f(\gamma)^{+}}^{V\left[G_{\gamma}\right]}$. For a review of revised countable support iterations, see [Fuc]. The difference between countable support and revised countable support does not cause any difficulties in the previous arguments, and the arguments easily adapt to the following:

\section{Theorem 13.}

(1) If $\kappa$ is strongly unfoldable and $0^{\sharp}$ does not exist, then the SPFA lottery preparation forces $\mathrm{SPFA}_{\mathfrak{c}}$, $\mathrm{SPFA}\left(\aleph_{2}\right.$-preserving) and $\mathrm{SPFA}\left(\aleph_{3}\right.$-preserving $)$, with $\mathfrak{c}=\kappa=\aleph_{2}$.

(2) If $\kappa$ is strongly unfoldable, then the SPFA lottery preparation forces $\mathrm{SPFA}_{\mathfrak{c}}$, $\operatorname{SPFA}\left(\aleph_{2}\right.$-covering $)$ and $\operatorname{SPFA}\left(\aleph_{3}\right.$-covering $)$, with $\mathfrak{c}=\kappa=\aleph_{2}$.

Corollary 14. The following are equiconsistent over ZFC:

(1) There is an unfoldable cardinal $\kappa$.

(2) $\operatorname{SPFA}\left(\aleph_{2}\right.$-preserving $)+\operatorname{SPFA}\left(\aleph_{3}\right.$-preserving $)+\mathrm{SPFA}_{\aleph_{2}}+2^{\omega}=\aleph_{2}$. 
(3) $\mathrm{SPFA}_{\mathrm{c}}$.

(4) $\operatorname{PFA}\left(\aleph_{2}\right.$-preserving $)+\operatorname{PFA}\left(\aleph_{3}\right.$-preserving $)+\mathrm{PFA}_{\aleph_{2}}+2^{\omega}=\aleph_{2}$.

(5) $\mathrm{PFA}_{\mathfrak{c}}$.

Our summary conclusion, viewing the unfoldable cardinals as relatively weak, is that in order to extract significant strength from PFA or SPFA, one must use forcing notions that collapse $\aleph_{3}$ to $\aleph_{1}$.

\section{REFERENCES}

[DH06] Mirna Džamonja and Joel David Hamkins. Diamond (on the regulars) can fail at any strongly unfoldable cardinal. Annals of Pure and Applied Logic, 144:83-95, December 2006. Conference in honor of the sixtieth birthday of James E. Baumgartner. MR 2279655 (2007m:03091)

[Fuc] Ulrich Fuchs. Donder's version of revised countable support. arXiv:math.LO/9207204.

[GS95] Martin Goldstern and Saharon Shelah. The bounded proper forcing axiom. J. Symbolic Logic, 60(1):58-73, 1995. MR1324501 (96g:03083)

[Ham] Joel David Hamkins. A class of strong diamond principles. arXiv:math.LO/0211419.

[Ham00] Joel David Hamkins. The lottery preparation. Ann. Pure Appl. Logic, 101(2-3):103-146, 2000. MR.1736060 (2001i:03108)

[Hau91] Kai Hauser. Indescribable cardinals and elementary embeddings. Journal of Symbolic Logic, 56:439-457, 1991. MR1133077 (92j:03049)

[HJ] Joel David Hamkins and Thomas A. Johnstone. Indestructible strong unfoldability, submitted.

[Joh] Thomas A. Johnstone. Strongly unfoldable cardinals made indestructible. Journal of Symbolic Logic, 73(4):1215-1248, 2008.

[Joh07] Thomas A. Johnstone. Strongly unfoldable cardinals made indestructible. Ph.D. thesis, The Graduate Center of the City University of New York, June 2007.

[Lav78] Richard Laver. Making the supercompactness of $\kappa$ indestructible under $\kappa$-directed closed forcing. Israel Journal of Mathematics, 29:385-388, 1978. MR0472529 (57:12226)

[Men74] Telis K. Menas. On strong compactness and supercompactness. Annals of Mathematical Logic, 7:327-359, 1974. MR0357121 (50:9589)

[Miy98] Tadatoshi Miyamoto. A note on weak segments of PFA. In Proceedings of the Sixth Asian Logic Conference (Beijing, 1996), pages 175-197, World Sci. Publishing, River Edge, NJ, 1998. MR1789737 (2001g:03092)

[Nee08] Itay Neeman. Hierarchies of forcing axioms, II. Journal of Symbolic Logic, 73:522-542, 2008. MR2414463

[NS08] Itay Neeman and Ernest Schimmerling. Hierarchies of forcing axioms, I. Journal of Symbolic Logic, 73:343-362, 2008. MR2387946

[Vil98] Andrés Villaveces. Chains of end elementary extensions of models of set theory. Journal of Symbolic Logic, 63(3):1116-1136, 1998. MR1649079 (2000h:03074)

Department of Mathematics, The Graduate Center of The City University of New York, 365 Fifth Avenue, New York, New York 10016 - and - Department of Mathematics, The College of Staten Island of The City University of New York, Staten Island, New York 10314

E-mail address: jhamkins@gc.cuny.edu

Department of Mathematics, New York City College of Technology of The City University of New York, 300 Jay Street, Brooklyn, New York 11201

E-mail address: tjohnstone@citytech.cuny.edu 